\title{
Cinquante ans d'études sur le moyen français dans les pays nordiques (1965-2015)
}

\section{Elina Suomela-Härmä et Juhani Härmä}

\section{(2) OpenEdition}

10 Journals

\section{Édition électronique}

URL : https://journals.openedition.org/studifrancesi/22074

DOI : $10.4000 /$ studifrancesi.22074

ISSN : 2421-5856

Éditeur

Rosenberg \& Sellier

\section{Édition imprimée}

Date de publication : 1 avril 2020

Pagination : 3-22

ISSN : 0039-2944

\section{Référence électronique}

Elina Suomela-Härmä et Juhani Härmä, « Cinquante ans d'études sur le moyen français dans les pays nordiques (1965-2015) », Studi Francesi [En ligne], 190 (LXIV | I) | 2020, mis en ligne le 01 avril 2021, consulté le 03 août 2021. URL : http://journals.openedition.org/studifrancesi/22074 ; DOI : https:// doi.org/10.4000/studifrancesi.22074

\section{(c) (i) $\odot$}

Studi Francesi è distribuita con Licenza Creative Commons Attribuzione - Non commerciale - Non opere derivate 4.0 Internazionale. 


\section{Cinquante ans d'études sur le moyen français dans les pays nordiques (1965-2015)}

\section{Abstract}

The article is a survey of the research done on Middle French (MF) in the Nordic countries during the period 1965-2015, a time of fruitful scholarship in that area. It starts with an examination of the use of the term Middle French in Nordic research, seldom used until the 1980s. The three subchapters concern a) text editions, b) literary research and c) linguistic studies. The twenty-one text editions published during those fifty years represent hagiographies and miracles, technical cynegetic and military treatises and inter- and intralingual translations. Literary research is scarce; not a single monograph entirely devoted to MF literature was published. The Danish journal "Revue Romane" is the publication responsible for the biggest amount of literary research, including reviews of recent publications. The linguistic subchapter presents monographs and other studies, representing diachronic and synchronic research. The first Nordic monograph on MF is Ulla Jokinen's Les relatives en moyen français (1978), which can be considered as the pioneer of MF linguistic research in Northern Europe. The central name in diachronic research is Lene Schøsler, whose studies often deal with the entire history of the French language since its Latin origins. Among the various topics in synchronic research, one can especially mention the studies concerning translations and translation techniques.

The contribution of Nordic scholars to MF research has been quite considerable, but this may no longer be the case in the future, since academic teaching and thus also research is more and more limited to contemporary French.

\section{Introduction}

Dans la Préface du volume Le Moyen Âge par le Moyen Âge, même qui recueille les contributions d'un séminaire organisé à l'Université de Stockholm en 2009, Jacqueline Cerquiglini-Toulet cite un article de Gaston Paris de 1888 dans lequel celui-ci souligne la vivacité des études philologiques en Suède ${ }^{1}$. Si on y regarde de plus près, on constate que le savant français, après avoir mentionné quelques philologues suédois, inclut dans sa présentation également la Finlande et la Norvège pour s'arrêter ensuite sur le Danemark où, dit-il, les études françaises sont particulièrement florissantes ${ }^{2}$. Cela démontre, si besoin est, que dans les pays nordiques la philologie française ne date pas d'hier, même si le présent survol, un bilan des études publiées entre 1960 et 2015, ne couvre qu'un demi-siècle. Notre point de départ est

(1) J. Cerquiglini-Toulet, Préface, in L. Brun et al. (éds), Le Moyen Âge par le Moyen Âge, même. Réception, relectures et réécritures des textes médiévaux dans la littérature française des XIV et XV siècles, Paris, Champion, 2015, «Colloques, congrès et conférences sur le Moyen Âge», pp. 9-10.

(2) G. Paris, Les cours d'amour du Moyen Âge, in M. Roques (publ.), Mélanges de littérature française du Moyen Âge, Paris, Honoré Champion, 1912, pp. 473-497; ici pp. 474-475. Danemark est ici synonyme de Université de Copenhague. 
en effet postérieur de quelque soixante-dix ans à celui de Gaston Paris, et, en outre, les considérations faites se limiteront aux travaux portant sur le moyen français ${ }^{3}$.

\section{Moyen français: terme et définitions}

Pour commencer, voici quelques remarques sur la présence du terme moyen français dans les études nordiques. Pendant bien longtemps et encore dans les années 1970, on parlait de préférence de l'ancienne langue en général, sans distinguer l'ancien du moyen français. Comme il allait presque de soi que les textes édités et les questions linguistiques débattues étaient relatifs aux XII ${ }^{\mathrm{e}}$ et $\mathrm{XIII}^{\mathrm{e}}$ siècles, une dénomination vague - telle que ancienne langue justement - était suffisante. Cependant on a une attestation précoce de moyen français déjà dans la Grammaire historique (1899) de Kristoffer Nyrop. Le linguiste danois propose de découper l'histoire du français en cinq périodes, dont celle qui est appelée moyenne s'étend d'après lui de la fin du $X V^{e}$ jusqu'à la fin du XVI ${ }^{e}$ siècle ${ }^{4}$. Cette définition est ensuite reprise telle quelle pendant plusieurs décennies, y compris dans le Précis historique de grammaire française (1974) de Knud Togeby. Pour voir ce qui se passe du côté des Suédois, nous avons dépouillé les 418 entrées de la bibliographie de Gunnar Tilander ${ }^{5}$ (1894-1973), un des premiers dans les pays nordiques à avoir édité des textes $\mathrm{du} \mathrm{XV}^{\mathrm{e}}$ siècle, autrement dit des textes dont la langue peut être qualifiée de moyen français, quels que soient les extrêmes chronologiques retenus. Le terme en question apparaît pour la première fois sous la plume de Tilander en 1936 - on le trouve dans le titre d'une étude lexicale - mais cette occurrence restera un hapax dans les intitulés de ses travaux. Jusqu'à ses dernières publications, Tilander recourt aux dénominations ancienne langue, vieux français et, bien que dans une moindre mesure, ancien français. On a beau chercher le terme moyen français même dans ses éditions de textes tardifs, y compris le Livre de Chasse de Gaston Phébus (1971), il ne s'y trouve pas. Ce n'est que dans l'introduction du Livre des oraisons du même Phébus, publié à titre posthume (1975), que Tilander constate que «[1] a langue de Gaston Phébus est en plein<e> période de transformation et de transition entre le vieux et le moyen français» ${ }^{7}$. Dans ce passage, moyen français s'oppose donc à vieux français, encore qu'aucune indication sur la délimitation de ces périodes ne soit donnée.

$\mathrm{Si}$, dès le début du $\mathrm{Xx}^{\mathrm{e}}$ siècle, quelques philologues finlandais se sont occupés de textes des $\mathrm{XV}^{\mathrm{e}}$ et $\mathrm{XVI}^{\mathrm{e}}$ siècles (voir note 53), eux non plus n'emploient pas moyen français dans les intitulés de leurs études. Nos lectures, à vrai dire fragmentaires, ne nous ont pas permis de relever ce terme dans leurs écrits. La première étude linguistique dans le titre de laquelle il se trouve, est la thèse de troisième cycle Expressions $d u$ commandement et du soubait en moyen français. Étude syntaxique basée sur les farces

(3) Nous tenons à remercier tous les collègues, notamment Anders Bengtsson, Hans Petter Helland, Lene Schøsler et Lars-Göran Sundell, qui nous ont fourni des indications bibliographiques précieuses.

(4) K. Nyrop, Grammaire historique de la langue française, t. I, Copenhague, Det nordiske Forlag, 1899, pp. $32-55$.

(5) H. Bohrn (éd.), Gunnar Tilander. Publications 1918-1973. Bibliographie établie en l'honneur du $50^{\text {ème }}$ anniversaire de sa soutenance de thèse, Stockholm, Kungliga Biblioteket, 1973, «Acta Bibliothecae Regiae Stockholmiensis».

(6) G. Tilander, Moyen français etoire petite lunaire (Botrychium lunaria), "Romania" LXII, 248, 1936, pp. 376-378.

(7) G. Phébus, Livre des oraisons. Les prières d'un chasseur, éd. avec introduction, glossaire et reproduction d'une miniature du ms. 616 de la Bibliothèque nationale de Paris par G. Tilander, Karlshamn, 1975, «Cynegetica», p. 18. 
et sotties des XV et XVI siècles ${ }^{8}$ (1959) d'Ulla Jokinen. Le terme, non discuté, n'apparaît cependant pas dans le corps du texte.

La tendance à éviter moyen français au détriment d'autres dénominations, comme $d u X I V^{e}$ ou $d u X V^{e}$ siècle, se manifeste pendant longtemps aussi dans les revues nordiques'. Les quelques occurrences antérieures aux années 1970-1980 se trouvent surtout dans des comptes rendus et sont dues à un effet d'écho: le terme, employé par un chercheur non-nordique, passe dans les commentaires concernant ses travaux ${ }^{10}$. Il est intéressant de constater que les premiers articles dont le titre mentionne ce terme apparaissent dans les mêmes années aussi bien en Suède (1980) qu'en Finlande (1981) et au Danemark (1981), d'abord en anglais (Middle French) et ensuite aussi en français.

Si le terme moyen français peine à entrer dans l'usage même parmi les spécialistes, on remarque aussi une certaine indifférence lorsqu'il s'agit de le définir. Les éditeurs l'emploient souvent avec une certaine désinvolture. Lene Schøsler revient à ce problème lors d'une introduction faite à une table ronde portant sur «les problèmes concernant le moyen français» qui date de $1985^{11}$. Elle propose quelques thèmes de discussion, à commencer par sommes-nous d'accord sur les limites temporelles du moyen français? et finit par une provocation en posant la question de savoir s'il est nécessaire de périodiser, puisqu'il en résulte parfois qu'en imposant des limites temporelles on sépare des faits liés entre eux. Ces questions sont toujours d'actualité.

\section{Les éditions de textes}

\section{Choix des textes à éditer}

Le premier aspect qui retiendra notre attention est le choix des textes à éditer. Dans la première moitié du $\mathrm{Xx}^{\mathrm{e}}$ siècle en Suède et en Finlande se sont formées des équipes de philologues, qui ont conjugué leurs efforts pour publier des œuvres d'un même auteur ou appartenant à un genre donné. Ainsi les Suédois ont-ils une longue tradition, toujours vivace, dans l'édition des vies de saints et d'autres contes pieux qui s'étalent du XII ${ }^{\mathrm{e}}$ au XV ${ }^{\mathrm{e}}$ siècle. Pendant le demi-siècle dont il est question ici, cette série s'est enrichie de plusieurs textes tant en ancien qu'en moyen français. Les traités cynégétiques ont aussi suscité un vif intérêt en Suède, mais il serait exagéré de parler à ce sujet d'«école», la personne de Gunnar Tilander ayant plus ou moins écrasé les deux autres aspirants cynégètes (Holmér; Wistedt ${ }^{12}$ ). Quoi qu'il en soit, les Suédois ont édité des dizaines de textes cynégétiques non seulement en français, mais aussi en latin, italien, espagnol et moyen anglais. La collection Cynegetica, destinée à recueillir

(8) L'étude, inédite, est déposée à la Bibliothèque universitaire de Helsinki.

(9) "Neuphilologische Mitteilungen" (1899-) en Finlande; "Studia neophilologica" (1928-) en Suède et "Revue romane" (1966-) au Danemark. Par rapport à moyen anglais, omniprésent dans les "Neuphilologische Mitteilungen" et "Studia neophilologica", l'apparition de moyen français dans nos revues est bien plus tardive.

(10) Voir les comptes rendus d'A. Långfors ("Le Moyen Âge” 32, janvier-avril, 1922, pp. 111-112) et de G. Holmér ("Studia neophilologica" 2, 1966, pp. 347-348).

(11) L. Schøsler, Problèmes concernant le moyen français. Table ronde sur le moyen français, in P. Mänttäri, E. Suomela-Härmä, O. Välikangas (éds), Communications relatives aux groupes de travail, $9^{e}$ Congrès des Romanistes Scandinaves. Publications du Département des Langues Romanes 5, Helsinki, Université de Helsinki, 1985, pp. 13-19.

(12) R. Wistedt préparait une édition du Livre de fauconnerie de Jean de Fransières, mais n'en a publié qu'un fascicule d'introduction de 33 pages, intitulé Le Livre de fauconnerie de Jean de Fransières, l'auteur et ses sources, Lund, Kungl. Vitterhets Historie och Antikvitets Akademien, 1967, «Filologiskt arkiv». 
les travaux de son fondateur, ne compte pas moins de dix-neuf titres, dont les deux ouvrages de première importance de Gaston Phébus, le Livre de chasse et le Livre des oraisons s $^{13}$. À l'heure actuelle, les philologues suédois continuent à s'occuper de textes hagiographiques; en outre, ils s'intéressent aux chroniques, aux mises en prose et aux traductions, genres qui d'ailleurs peuvent s'entrecouper. En ce qui concerne la Finlande, l'activité de l'équipe travaillant sur les miracles de Gautier de Coinci (ca. 1937-1963) est restée isolée dans son genre. Les philologues des générations suivantes n'ont pas eu de centres d'intérêt communs à part une édition collective d'un Végèce français anonyme de $1380(1989)^{14}$. Comme leurs collègues suédois, les philologues finlandais s'intéressent aux traductions et aux mises en prose, devenues d'actualité pendant ces dernières décennies. Quant au Danemark et à la Norvège, ils sont peu présents dans ce premier volet de notre étude, non pas qu'il n'y ait pas de philologues, mais parce que les données chronologiques de leurs éditions ne coïncident pas avec celles que nous nous sommes fixées.

La qualité littéraire de la plupart des textes édités, de nature édifiante, didactique ou allégorique, reste en général médiocre. En revanche, ils sont d'autant plus intéressants comme témoins linguistiques, teintés ou non de traits dialectaux. La moitié des textes publiés a été transmise par un ou deux témoins, alors que le Livre de chasse de Gaston Phébus et le Traittié de conseil de Guillaume Fillastre sont conservés respectivement dans 44 et 13 manuscrits (cf. ci-dessous). Parmi les 21 éditions répertoriées, une seule, Le Plait de Brisebare, se base sur un ms. conservé dans une bibliothèque nordique, la Bibliothèque royale de Copenhague. Il est vrai que dans celles-ci les mss. médiévaux français n'abondent pas, mais rappelons tout de même que la collection Thott (Bibliothèque royale de Copenhague) possède entre autres un remarquable chansonnier français du XIV siècle (Thott 291, $8^{\circ}$ ) et la Bibliothèque royale de Stockholm, un ms. de Villon (Vu22). La majorité des textes édités, 13 sur 21 , est publiée pour la première fois (abstraction faite des imprimés du XIV siècle). Un sous-chapitre du type Pourquoi éditer X?, comme celui inclus dans l'introduction du Traittié de conseil de Guillaume Fillastre ${ }^{15}$, rendrait service aux lecteurs non familiers de textes anciens.

Éditions, manuscrits, datations

Rester dans les limites chronologiques conventionnelles (1330 ou $1350 / 1500)^{16}$ nous crée bien des difficultés tant en aval qu'en amont: une question aussi simple que le nombre des éditions publiées entre 1965 et 2015 suffit à nous mettre dans $l^{\prime}$ embarras ${ }^{17}$. Comme certains textes ne sauraient être datés qu'avec approximation par ex. du premier tiers du XIV siècle - ils restent dans une zone grise problématique. Le panorama est en outre brouillé par les textes dont la datation est sujette à des controverses (cf. ci-dessous).

(13) Les références bibliographiques des éditions sont données dans la note 19.

(14) Cependant, l'“équipe Golein” prépare sous la direction d'O. Merisalo une édition de la traduction de Jean Golein de De informacione principum. Cf. O. Merisalo, Jean Golein, clergonnet du roy. Observations sur la traduction du De informacione principum attribuée à Golein, in J. Ducos et M. Goyens (éds), Traduire au XIV siècle: Evrart de Conty et la vie intellectuelle à la cour de Charles V, Paris, Champion, 2015, «Colloques, congrès et conférences, sciences du langage, histoire de la langue et des dictionnaires», pp. 327-336.

(15) Voir note 13.

(16) Conventionnelles en ce sens que 1330 et 1500 sont les limites fixées par le DMF.

(17) Ne seront prises en considération que les publications monographiques. Les éditions parues dans des revues sont d'ailleurs rarissimes. 
Pour présenter notre "corpus" d'éditions, nous avons finalement opté pour la solution suivante: sera d'abord signalé un premier groupe de textes (A) dont la datation s'échelonne entre 1330 et 1350 . Ensuite sera présenté moins sommairement un second groupe (B), celui des textes datables entre 1350 et 1510, dont l'appartenance à la "période moyenne" ne saurait être contestée. On notera qu'à une exception près, les textes tardifs (seconde moitié du Xv ${ }^{\mathrm{e}}$ siècle) ont paru entre 1994 et 2017 (bien que sa date de parution soit postérieure à 2015, le texte de Jean Servion a été inclus dans notre corpus étant donné sa «parenté» avec Theseus de Cologne). La table suivante offre une vue d'ensemble des éditions des deux groupes (la date de parution se trouve entre parenthèses):

\begin{tabular}{|c|c|}
\hline $\begin{array}{c}\text { A } \\
\text { Textes datés de 1330-1350 }\end{array}$ & $\begin{array}{c}\text { B } \\
\text { Textes datés de 1350-1510 }\end{array}$ \\
\hline 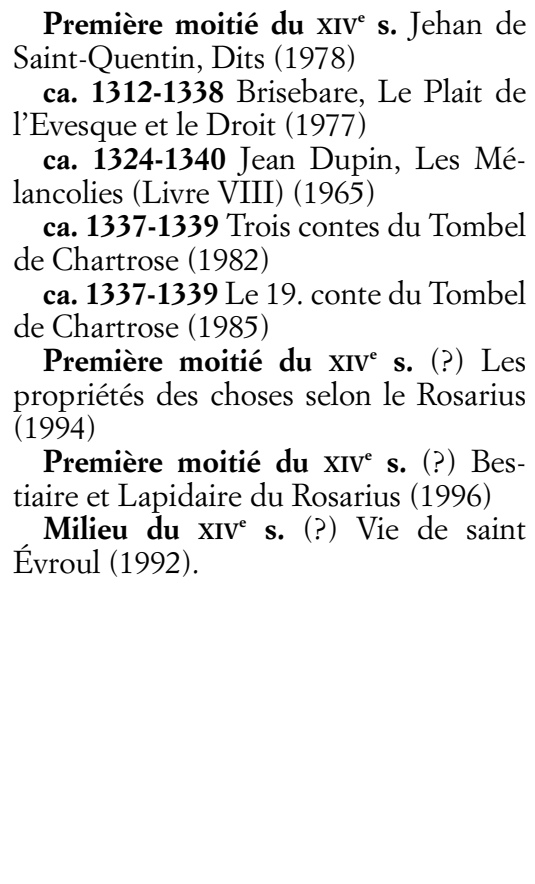 & $\begin{array}{l}\text { ca. 1360-xve s. Vie de sainte Gene- } \\
\text { viève (2006) } \\
\text { 1380 Vegesce, Le Livre de l'art de } \\
\text { chevalerie (traduction anonyme) (1989) } \\
\text { 1380 Gaston Phébus, Livre des Orai- } \\
\text { sons (1975) } \\
\text { 1387-1389 Gaston Phébus, Livre de } \\
\text { chasse (1971) } \\
\text { ca. 1388 Jean de Noyal, Miroir histo- } \\
\text { rial (2012) } \\
\text { après } 1378 \text { Theseus de Cologne } \\
\text { (2008) } \\
\text { Xv's. Vie de sainte Bathilde (1996) } \\
\text { ca. 1450 Jean Miélot, Vie et miracles } \\
\text { de Saint Josse (2004) } \\
\text { ca. } 1460 \text { Le Livre de Regnart (mise en } \\
\text { prose de Renart le Nouvel) (1998) } \\
\text { ca. 1466 Jean Servion, mise en prose } \\
\text { du Théséus de Cologne (2017) } \\
\text { 1472-1473 Guillaume Fillastre, Le } \\
\text { Traittié de conseil (1994) } \\
\text { 1483 }{ }^{18} \text { Le Débat du Faucon et du } \\
\text { Lévrier. Traduction de Robert du Herlin } \\
\text { (1978) } \\
\text { ca. 1504 Pétrarque, Les Triomphes. } \\
\text { Traduction de Simon Bourgouin (2012). }\end{array}$ \\
\hline
\end{tabular}

Table I. Éditions de textes en moyen français dans les pays nordiques (1965-2015) ${ }^{19}$.

(18) Cette datation a été proposée par J.-Y. Badel, Le Pommier de douceur de Robert du Herlin (1481), in Remembrances et Resveries, Hommage à Jean Batany, Orléans, Éditions Paradigme, 2006, «Medievalia». pp. 71-83; ici p. 73.

(19) Dits en quatrains d'alexandrins monorimes de Jehan de Saint-Quentin, éd. par B. Munk Olsen, Paris, A. et J. Picard et Cie, 1978, SATF; Brisebare, Le plait de l'Evesque et de Droit, éd. critique par J. Kjær, "Revue romane" numéro spécial, 1977; Les mélancolies de Jean Dupin, éd. critique par L. Lindgren, Turku, Annales universitatis Turkuensis, series B, tom. 95, 1965; Trois contes français du XIV siècle tirés du recueil intitulé "Le tombel de Chartrose", publiés avec introduction, notes et glossaire par S. Sandqvist, Stockholm, 1982, «Acta Universitatis Lundensis. Sectio I. Theologica juridica humaniora»; La mort du roi Souvain. Le 
Les textes du groupe $\mathrm{A}$

L'ouvrage mis en tête du groupe A est attribué à un certain Jehan de Saint-Quentin, actif dans la première moitié du XIV ${ }^{e}$ siècle, dont on ne connait que le nom. On lui doit l'ensemble ou une partie des vingt-deux Dits préservés dans le ms. BnF fr. 24432, dont quatorze avaient déjà été inclus dans le Nowveau recueil de contes d'A. Jubinal (1839). Le titre de l'ouvrage de Brisebare a été reformulé par l'éditrice. Le ms. (Bibliothèque royale de Copenhague, ancien fonds royal 2061-4 $)$ donne en effet (Chi commence) De l'Evesque de Droit, transformé par J. Kjær en Le Plait de l'Evesque et le Droit. Le titre ne laisse pas deviner qu'il s'agit d'une psychomachie. Les Mélancolies de Jean Dupin ont été dépouillées pour le Dictionnaire du Moyen Français $(D M F)$, mais comme la déclinaison des noms est respectée dans certains des mss., l'éditeur qualifie l'auteur d'«écrivain de l'ancien français» ${ }^{20}$. L'édition du livre VIII des Mélancolies, publié pour la première fois, se base sur le ms. 586 de la Bibliothèque municipale de Besançon. Moitié état du monde, moitié traité moralisant, ce texte n'a pas eu autant de succès que la première partie de l'ouvrage: il ne se trouve que dans neuf des seize manuscrits.

Si Sven Sandqvist a choisi de rééditer, dans deux volumes séparés, certains des contes dévots (numéros 19; 1, 2 et 3) du recueil Tombel de Chartrose, c'est tout d'abord parce que celui dont le héros est le roi Souvain (numéro 19) - par ailleurs un personnage de l'histoire du Danemark - n'était disponible que dans une édition diplomatique de 1846. Et comme le projet caressé par son compatriote Emmanuel Walberg de publier l'ensemble des 31 contes ne s'est réalisé que partiellement ${ }^{21}$, Sandqvist a jeté son dévolu sur les trois premiers, exclus des éditions walbergiennes. Son édition était déjà bien avancée, lorsqu'est sortie celle du néerlandais E. Kooimans $(1975)^{22}$ qui comprend entre autres les trois premiers contes. Sandqvist n'en a

dix-neuvième conte du recueil intitulé "Le tombel de Chartrose", publié avec introduction, notes et glossaire par S. Sandqvist, Stockholm, 1985, «Kungl. Vitterhets historie och antikvitets akademien, Filologiskt arkiv»; Les propriétés des choses selon le "Rosarius" (B.N. f. fr. 12483), publ. par A. Zetterberg, éd. revue et complétée par S. Sandqvist, Lund, 1994, «Études romanes de Lund»; Le bestiaire et le lapidaire du "Rosarius" (B.N.f.fr. 12483), éd. par S. Sandqvist, Lund, 1996, «Études romanes de Lund»; La vie de saint Évroul, publié avec introduction, notes et glossaire par S. Sandqvist, Lund, 1992, «Études romanes de Lund»; La Vie de sainte Geneviève. Cinq versions en prose des XIV et XV siècles, éd. par A. Bengtsson, Stockholm, 2006, «Acta Universitatis Stockholmiensis»; Le Livre de l'Art de chevalerie de Vegesce. Traduction anonyme de 1380, éd. par L. Löfstedt et al., Helsinki, Suomalainen Tiedeakatemia, 1989; Gaston Phébus, Livre des oraisons. Les prières d'un chasseur cit.; Gaston Phébus, Livre de chasse, éd. avec introduction, glossaire et reproduction des 87 miniatures du ms. 616 de la Bibliothèque nationale de Paris par G. Tilander, Karlshamn, 1971, «Cynegetica»; Jean de Noyal, Miroir historial. Livre X, éd. critique par P. Förnegård, Genève, Droz, 2012, «TLF»; Theseus de Cologne, édition partielle d'une chanson de geste du XIV siècle, éd. par M. Bacquin, Lund, 2008, «Études romanes de Lund»; La Vie de Sainte Bathilde: quatre versions en prose des XIII et XV siècles, publ. avec introduction, notes et glossaire par A. Bengtsson, Lund, 1996, «Études romanes de Lund»; Jean Miélot, Vie et miracles de Saint Josse, publ. avec introduction, notes et glossaire par N.-O. Jönsson, Turnhout, Brepols, «Textes vernaculaires du Moyen Âge », 2004; Le Livre de Regnart, éd. critique avec introduction, notes et glossaire par E. Suomela-Härmä, Paris, Champion, 1998, «Bibliothèque du Xv'siècle»; Le Théséus de Cologne de Jean Servion - un cri au secours, publ. par M. Bacquin, Lund, 2017, «Études romanes de Lund»; Guillaume Fillastre, Le Traittié de Conseil, éd. critique avec introduction, commentaire et glossaire par H. Häyrynen, Jyväskylä, 1994, «Studia philologica jyväskyläensia»; Le Débat du Faucon et du Levrier, éd. par G. Holmér, Stockholm, 1978, «Romanica Stockholmiensia»; Pétrarque, Les Triomphes. Traduction française de Simon Bourgouin, éd. critique, introduction et notes par G. Parussa et E. Suomela-Härmä, Genève, Droz, 2012, «Travaux d’Humanisme et Renaissance».

(20) L. Lindgren, Les Mélancolies cit., pp. 17-18 et p. 28.

(21) Après avoir publié trois de ces contes dans différents mélanges et revues, E. Walberg en a édité encore neuf autres sous le titre Contes pieux en vers $d u$ XIV siècle tirés du recueil intitulé "Le tombel de Chartrose", Lund, 1946, «Skrifter utgivna av Kungl. Humanistiska Vetenskapssamfundet i Lund».

(22) Nous n'avons pas pu consulter cette édition. Selon A. Gier, "Vox Romanica" 42, 1983, p. 331, elle s'intitule 18 contes français tirés du recueil intitulé "Le Tombel de Chartrose" édités avec variantes et précédés d'une introduction et a été publiée à Amsterdam en 1975. 
pas pour autant renoncé à son entreprise, puisqu'il lui semblait qu'il restait encore des questions à élucider ${ }^{23}$. On peut regretter que les quatre histoires n'aient pas été éditées ensemble, d'autant plus qu'elles se basent sur le même ms. (Bibl. Municipale d'Avranches 244). En principe, on pourrait en dire autant des chapitres tirés du Rosarius, une compilation faite en l'honneur de la Vierge (BnF fr. 12483), si ce n'est que dans ce cas-ci, les éditeurs sont au nombre de deux. Comme Anders Zetterberg $(\dagger 1992)$ n'a pas pu parachever son édition de la partie intitulée Les propriétés des choses, Sandqvist y a mis la dernière main; ensuite, fort de cette expérience, il a affronté deux autres textes encore inédits du recueil, le bestiaire et le lapidaire.

Arrêtons-nous maintenant pour examiner brièvement la datation des deux derniers textes du groupe $A^{24}$. Sandqvist propose de repousser de deux cents ans celle du poème normand Vie de Saint Evroul (BnF f. fr. 19867) (25 $^{25}$ et qualifie la langue du Rosarius plutôt de moyen que d'ancien français ${ }^{26}$. Cela faisant, il s'oppose résolument à l'avis exprimé par Arthur Långfors (1881-1959) plusieurs décennies plus tôt. Le savant finlandais a en effet écrit un compte rendu, par ailleurs peu flatteur, de l'édition de F. Danne de la Vie de saint Évroul ${ }^{27}$; on lui doit en outre une étude approfondie sur le ms. BnF fr. 12483 contenant le Rosarius ${ }^{28}$.

Voici d'abord des arguments pour la datation de Saint Évroul: selon les deux premiers éditeurs du poème, J.B.N. Blin (1887) et F. Danne (1913), celui-ci remonterait à la seconde moitié du XII ${ }^{\mathrm{e}}$ siècle. Cela n'est pas contesté par Långfors qui affirme laconiquement que le poème est probablement peu postérieur au milieu du $\mathrm{XII}^{\mathrm{e}}$ siècle, tandis que l'unique manuscrit remonte au XIV $\mathrm{Xiècle}^{29}$. Cependant, dans une notice lexicographique, J.-L. Ringelbach confond les deux datations et affirme que Långfors situe la Vie au XIV siècle $^{30}$. Contrairement à ce que fait Sandqvist, il faut donc ignorer cette conclusion hâtive: Långfors ne soutient pas une datation plus tardive. Quant aux événements historiques évoqués ou passés sous silence dans le poème, un exemple suffira: d'après Blin, le fait qu'il n'y soit pas question de la translation des reliques de Saint Evroul, survenue en 1214, signifie qu'il est antérieur à cette date. Or pour Sandqvist il est facile de démontrer qu'ici comme ailleurs, le poète ne fait que suivre sa source, Orderic Vital, dont l'histoire se termine en 1141. En ce qui concerne les traits linguistiques allégués, Sandqvist relève des formes comme ceci, cela, avant que, lequel (comme adj.) et si tost que au sujet desquelles il constate: «Cette dernière catégorie [de mots] est particulièrement intéressante pour la datation du texte. Il est en effet invraisemblable qu'un auteur

(23) S. Sandqvist, Tombel cit., p. 13.

(24) La datation des textes n'a pas été débattue seulement dans les introductions des éditions. Ainsi Povl Skårup revient-il sur celle du Miroir des simples âmes, texte attribué à Marguerite Porete et édité par Romana Guarnieri en 1986. Selon Skårup, et contrairement à ce qu'affirme l'éditrice, la langue de ce texte «n'est pas l'ancien français de la fin du XIII siècle, mais le moyen français d'après 1370» (La langue du "Miroir des simples âmes" attribué à Marguerite Porete, "Studia Neophilologica" 60, 2, 1988, pp. 231-236, ici p. 231). Tout comme Sandström, Skårup s'appuie sur des phénomènes lexicaux. Dans son compte rendu de l'édition en question, L. Löfstedt vient étayer indirectement l'avis de Skårup en constatant que le texte français de cette édition bilingue «doit intéresser un spécialiste du moyen français» (les italiques sont nôtres), "Neuphilologische Mitteilungen" XC, 2, 1989, p. 235.

(25) Saint Evroul cit., p. 35.

(26) Le bestiaire cit., p. 6.

(27) A. Långfors, c.r. de l'édition de F. Danne, "Romania” XLIV, 1915-1917, pp. 102-103.

(28) A. Långfors, Notice du manuscrit français 12483 de la Bibliothèque nationale. Tiré des Notices et extraits des manuscrits de la Bibliothèque nationale et autres bibliothèques, t. 39, 2. Paris, Imprimerie Nationale, 1916.

(29) A. Långfors, Compte rendu cit., p. 102

(30) Ringelbach prétend en effet que «la Vie de Saint Evroul ne doit pas être datée de 1180, mais du XIV ${ }^{e}$ s. (...) cf. A. Långfors ds Romania 44». TraLiPhi XXVIII, 1989, p. 14. 
du XII ${ }^{\mathrm{e}}$ siècle ait utilisé des mots grammaticaux qui apparaissent dans d'autres textes cent ans, parfois presque deux cents ans plus tard $\gg^{31}$.

Quant au Rosarius, les traits propres au mfr. identifiés par Sandqvist sont plus nombreux que dans la Vie de saint Évroul, mais l'enjeu est moindre: il ne s'agit que de repousser la datation traditionnelle (vers 1330) de quelques dizaines d'années. La question reste pourtant épineuse, car même au dire de Sandqvist, «aucun trait (c.à-d. morphosyntaxique) isolé ne réfute la datation proposée par Långfors [à savoir 1330]»; en outre, l'affirmation de l'éditeur selon laquelle le lexique «appartient aussi au moyen français» ne repose que sur une «impression» ${ }^{32}$. Par contre, les arguments avancés par Långfors se basent d'une part sur des faits extralinguistiques; de l'autre, il juge l'écriture du ms. 12483 «très peu postérieur[e]» à 1328 , date ante quem non ${ }^{33}$. Si le recours aux détails extralinguistiques peut ne pas convaincre, il reste que dans les deux cas les considérations codicologiques de Långfors, lapidaires et nullement explicitées, ne soutiennent pas les arguments d'ordre lexicologique de Sandqvist. Les datations en question mériteraient encore un nouvel examen.

\section{Les textes du groupe B}

La première édition de ce groupe, le Vegesce anonyme de 1380, clôt la série des traductions françaises de l'Epitoma rei militaris publiées par Leena Löfstedt. Après avoir édité les deux premières, dues à Jean de Meun (1977) et à Jean de Vignay (1982), elle a en effet proposé à un groupe de jeunes chercheurs de préparer sous sa direction une édition de la traduction la plus tardive (Bruxelles, Bibliothèque royale 11046). La datation de ce texte anonyme nous est donnée par les deux mss. connus, dont l'incipit contient la mention suivante: Ce traitié ... fut translaté ... l'an de grace mil CCC. IIIIXX.

Le Livre de chasse de Gaston Phébus, édité par deux fois au XIX siècle, est également un traité technique. Il va de soi que Tilander, passionné de chasse comme il l'était, ne pouvait pas ne pas rééditer ce chef-d'œuvre de cynégétique, ne serait-ce que parce que Phébus est venu en Suède et y a chassé le renne, animal qu'il n'avait pas vu en romain païs. Après avoir énuméré les 44 mss. de sa connaissance ${ }^{34}$, il en retient quatre, le ms. de base ${ }^{35}$ et trois autres qui lui servent pour la collation. Dans le commentaire qu'il fait de celui-là, Tilander se limite à renvoyer au catalogue d'A. de Laborde qui en donne une description moins sommaire ${ }^{36}$. On aimerait savoir sous quelle forme Tilander a consulté le ms. saint-pétersbourgeois: s'est-il servi d'un microfilm ou de photos, s'est-il rendu sur place pour le copier ou l'a-t-il fait venir à Stockholm ${ }^{37}$ ? Quoi qu'il en soit, au lieu de décrire lui-même le ms., Tilander promet

(31) S. Sandqvist, Évroul cit. p. 34.

(32) A. Zetterberg et S. Sandqvist, Les propriétés cit., pp. 44-45.

(33) A. Långfors, Notice cit., p. 7. P. Kunstmann retient aussi la datation de Långfors, cf. Miracles de Notre-Dame, tirés du Rosarius (Paris, ms. B.N. fr. 12483), Ottawa-Paris, Les Presses de l'Université d'Ottawa, 1991, «Publications médiévales de l'Université d'Ottawa», p. I.

(34) Deux autres exemplaires ont encore réapparu par la suite, cf. Fr. Avril, Les quatre mss. principaux L, D, A et P, in Gaston Phébus, Das Buch der Jagd. Ms M. 1044, The Pierpont Morgan Library, New York. Commentaire, Vol. 2, Fr. Avril et W.M. Voelkle, Editions Facsimilé, Lucerne, pp. 135-157.

(35) Le ms. en question est conservé actuellement à la Bibliothèque nationale de Russie à Saint-Pétersbourg sous la cote OP $\mathrm{N}^{\circ} 2$.

(36) A. de Laborde, Les principaux manuscrits à peintures conservés dans l'ancienne Bibliothèque impériale de Saint Pétersbourg, II, "Bulletin de la Société française de reproduction de manuscrits à peintures" 22, 1938, p. 175.

(37) Le prêt à distance d'un ms. ancien nous semble aujourd'hui inimaginable, mais nous connaissons un précédent: en 1932, la Bibliothèque de Saint-Pétersbourg (à l'époque Leningrad) avait envoyé un ms. du XII ${ }^{e}$ s. de Gautier de Coinci à la Bibliothèque universitaire de Helsinki, où Långfors a pu le consulter à sa guise pendant trois mois ("Suomen Kuvalehti" 51-52, 1932, pp. 1840-1841). 
de publier plus tard un volume palliant cette lacune et offrant aussi «la riche floraison des variantes des manuscrits» ${ }^{38}$ non retenues. Il n'a cependant pas eu le temps de parachever ce travail qui aurait dû être le numéro 20 de la collection Cynegetica. Ainsi le lecteur ignorera-t-il entre autres que l'édition ne respecte pas partout l'ordre dans lequel les chapitres se suivent dans le ms. de base ${ }^{39}$. Quant au Livre des Oraisons, datable avec précision, puisqu'il a été écrit immédiatement après les événements tragiques auxquels il est lié, il est publié d'après le même ms. saint-pétersbourgeois. Dans les six témoins qui contiennent ce texte, il est en effet précédé ou suivi du Livre de chasse.

À une exception près, les versions inédites en prose des Vies de Sainte Bathilde et de Sainte Geneviève, publiées par Anders Bengtsson, ont été transmises par plusieurs ms. Dans sa première édition (Sainte Bathilde), l'éditeur se limite à dater les mss. et ne dit mot sur l'époque de la rédaction des textes. Les Vies de Sainte Geneviève sont par contre situées dans le temps. D'après Bengtsson, l'excellente qualité de la "version II" pourrait permettre une nouvelle identification du traducteur, qui serait non pas Thomas Benoist, comme on l'a cru jusqu'à maintenant, mais Jean Golein. Bengtsson constate à ce propos que «s'il faut choisir entre un Thomas Benoist totalement inconnu, dont on ne trouve le nom nulle part dans les manuscrits, et un Jean Golein, traducteur prolifique, notamment d'hagiographies, dont le nom est attesté dans un des manuscrits $(. .$.$) , la seconde hypothèse nous semble plus crédibles { }^{40}$. Lorsque Nils-Olof Jönsson se mit à préparer une édition de Vie et Miracles de Saint Josse de Jean Miélot, il avait déjà à son actif l'édition d'une Vie de Saint Josse par Pierre de Beauvais ${ }^{41}$. Le texte de Miélot (Bruxelles KBR 10958) est considéré comme autographe; il en subsiste une copie à la Bibliothèque municipale de Valenciennes (ms. 511). D'après Doutrepont, l'œuvre est «en partie traduite, en partie originale» ${ }^{42}$. L'édition de Jönsson reste pour le moment la dernier des vies de saints publiées par les philologues suédois.

Le Roman de Renart a survécu aux XIV et $\mathrm{XV}^{e}$ siècles grâce à des épigones dont le dernier est Le Livre de Regnart, la mise en prose du Renart le Nowvel de Jacquemart Gielée. Une fois ce texte édité, on peut se faire une idée complète des usages multiples auxquels la matière renardienne s'est pliée au cours des siècles. L'auteur anonyme du Livre de Regnart ${ }^{43}$, lui, a "farci" le dérimage de 34 exemples, puisés principalement dans le Livre de Bonnes Moeurs de Jacques Legrand et se rattachant tant bien que mal au chapitre qu'ils sont censés commenter. Le héros éponyme de la chanson de geste Théséus de Cologne dont Mari Bacquin a publié un extrait inédit de presque quatre mille vers (BnF nouv. acq. fr. 10060) réapparaît lui aussi dans un contexte inattendu, à savoir le prologue des Chroniques de Savoie de Jean Servion. Les vicissitudes narrées dans la partie éditée de la geste ne sont pas celles auxquelles s'intéresse Jean Servion: celle-là concerne les aventures des petits-fils de Théséus, tandis que Servion raconte comment il se fait que ce dernier soit un ancêtre lointain des ducs de Savoie. Pour rétablir le texte de Servion, dont le seul ms. a péri dans l'incendie de la bibliothèque

(38) G. Tilander, Chasse cit., p. 27.

(39) Fr. Avril, Les quatre manuscrits cit., p. 137, note 6.

(40) A. Bengtsson, La Vie de Sainte Geneviève cit., pp. X-XI.

(41) N.-O. Jönsson, La Vie de saint Germer et la Vie de Saint Josse par Pierre de Beauvais. Deux poèmes du XIII ${ }^{e}$ siècle, publiés avec introduction, notes et glossaire. Lund, 1997, «Études romanes de Lund».

(42) G. Doutrepont, La littérature française à la cour des Ducs de Bourgogne. Paris, 1909, Champion, «Bibliothèque du Xve siècle», p. 214.

(43) Le texte est conservé dans un seul ms. (Chantilly, Mus. Condé, 473) et quatre éditions plus ou moins complètes du XVI ${ }^{\mathrm{e}}$ siècle. 
de Turin en 1904, Bacquin a entrepris une opération délicate, la reconstitution du texte en question à partir des deux éditions du XIX siècle, l'une collective (1840), l'autre due à F.E. Bollati (1879).

En choisissant d'éditer une partie du Miroir historial (ca. 1388) de l'abbé bénédictin Jean de Noyal, Per Förnegård vise à contribuer «aux recherches menées sur les compilations» et à enrichir les trop rares éditions de chroniques universelles des XIV ${ }^{\mathrm{e}}$ et $\mathrm{XV}^{\mathrm{e}}$ siècles ${ }^{44}$. Des trois livres (X-XII) conservés dans l'unique ms. (BnF f. fr. 10138), Förnegård publie le premier couvrant les années 1223-1328, resté pratiquement inédit jusqu'à nos jours. L'étonnante hétérogénéité de la langue de cette chronique s'explique par le fait que l'auteur puise à pleines mains dans ses sources $(74 \% \mathrm{du}$ livre dix provient de la Chronique amplifiée des rois de France) sans se soucier d'uniformiser son texte. L'édition préparée par Helena Häyrynen du Traittié de Conseil de Guillaume Fillastre a ceci en commun avec celle de Förnegård qu'il s'agit de la publication partielle d'une compilation faite par un bénédictin. On est étonné d'apprendre que c'est le premier texte français de Guillaume Fillastre à être édité. Fillastre fut en effet un personnage important à la cour de Philippe le Bon, dont le fils, Charles le Téméraire, lui commanda l'Histoire de la Toison d'or (le Traittié en est une partie). La plupart des treize mss. contenant le Traittié ont appartenu à des familles princières (le texte est en fait une sorte de miroir des princes) et le ms. de base de l'édition (Vienne, Archives de la Toison d'Or, 2) a été dédié au Téméraire.

Les deux textes les plus tardifs de ce survol sont des traductions inédites, l'une du latin, l'autre de l'italien, dans lesquelles le texte de départ a été copié dans la marge. Celui-ci peut ne pas coïncider (Débat) ou coïncider (Triomphes) avec le texte que le traducteur avait sous les yeux. Dans le seul ms. contenant la traduction du Débat du Faucon et du Lévrier (BnF fr. 1995) par Robert du Herlin, le texte source latin présente d'ailleurs des lacunes que l'éditeur Gustaf Holmér a comblées à l'aide d'un autre ms. latin. Signalons en passant l'analyse traductologique faite par Holmér ${ }^{45}$ à une époque où ce genre de commentaires n'était pas encore fréquent. Le texte italien de Pétrarque, provenant de l'édition de B. Zani (Venise, 1500) ${ }^{46}$, est reproduit en entier dans trois des cinq mss. français, dont le ms. de base de l'édition (BnF fr. 12423). Cela permet de vérifier que plusieurs des "bévues" du traducteur sont dues à un texte de départ imparfait. En s'efforçant de rendre le poème italien en alexandrins français, Simon Bourgouin apporte une contribution non dépourvue d'intérêt à la nouvelle langue poétique qui va se forger au $\mathrm{XVI}^{\mathrm{e}}$ siècle.

\section{Paratextes; méthodes d'éditions}

Les principaux changements subis par les paratextes pendant la période examinée concernent tout d'abord la description codicologique du ms. Dans les éditions plus anciennes, on se limitait à dater le ms. et éventuellement à commenter son état actuel. Ce n'est que dans un deuxième temps que s'est généralisée l'habitude de décrire le codex avec une terminologie adéquate. Les tentatives d'élaborer des stemmas sont pratiquement inexistantes. La méthode d'édition unanimement adoptée est celle du "meilleur" manuscrit; la tradition manuscrite des textes se réduit d'ailleurs souvent à un ou deux témoins. Les analyses linguistiques suivent le schéma traditionnel (voyelles, consonnes...) avec l'exception du Miroir historial qui

(44) P. Förnegård, Miroir cit., p. 19.

(45) G. Holmér, Débat cit., pp. 22-26.

(46) G. Parussa et E. Suomela-Härmä, Triomphes cit., p. 20. 
a poussé l'éditeur à publier à part un fascicule sur les démonstratifs dans le texte en question ${ }^{47}$. Les glossaires ont été révolutionnés. L'existence de bases de données comme le $D M F$ a fortement contribué à améliorer la présentation des lemmes. En outre, l'élaboration des concordanciers ne présentant plus de difficultés particulières, les éditeurs disposent de données difficilement accessibles par les méthodes traditionnelles. Les glossaires qui n'expliquent pas le choix des lemmes ou ignorent les mots inconnus à l'éditeur appartiennent désormais au passé. Au contraire, on vise à une description aussi exhaustive que possible de la matière lexicale; Per Förnegård est allé jusqu'à recenser «tous les mots (lemmes et formes fléchies) attestés dans le texte édité» ${ }^{48}$. Or si une telle prouesse n'était pas à la portée des chercheurs qui travaillaient sans le concours d'outils électroniques, ceux-ci avaient toutefois d'autres moyens pour arriver à des résultats remarquables. Après une vie consacrée aux recherches lexicales, un Tilander peut constater avec un orgueil justifié que «[1]e glossaire [du Livre de Chasse de Gaston Phébus] est le fruit de plus de quarante ans de recherches, au cours desquels j'ai dépouillé systématiquement tous les grands auteurs cynégétiques français» ${ }^{49}$.

La qualité des éditions est en général bonne et, dans quelques cas, très bonne. On peut certes toujours formuler des observations critiques; du point de vue des lecteurs, il est loisible de regretter le fait, en soi compréhensible, que certains textes au volume imposant ne soient édités que partiellement, voire par des éditeurs différents. Ainsi, qui veut se faire une idée de la totalité des textes du Rosarius, doit réunir des éditions publiées dans différents pays et séparées par des intervalles de plusieurs décennies ${ }^{50}$. Une autre difficulté matérielle à laquelle le lecteur se trouve confronté est que certains éditeurs tendent à renvoyer à leurs travaux antérieurs plutôt que de répéter une explication donnée ailleurs. Dans le pire des cas, la parfaite compréhension de la description d'un phénomène morphosyntaxique ou lexical nécessite ainsi la consultation simultanée de plusieurs publications, souvent parues dans des collections confidentielles.

Dans un article de $2000^{51}$, van Reenen et Schøsler fournissent une réflexion intéressante sur la manière dont une édition critique devrait se présenter pour que le lecteur ait à sa disposition toutes les informations possibles sur le texte édité et sa tradition manuscrite. Dans ce qu'ils appellent leur «credo méthodologique», ils définissent les quatre niveaux que cette édition idéale devrait comprendre $e^{52}$. On souscrit volontiers à ces propositions ambitieuses, mais leur réalisation, qui nécessite entre autres la contribution d'informaticiens, n'est guère à la portée d'un philologue travaillant seul.

(47) P. Förnegård, Les démonstratifs dans une compilation bistorique du XIV siècle. Préfixation, microsystèmes, cooccurrences, Stockholm, 2012, Sällskapet Runica et Mediaevalia, «Runica et Mediævalia. Opuscula».

(48) Id., Miroir cit., p. 377.

(49) G. Tilander, Chasse cit., p. 45.

(50) Rien qu'en Suède, trois philologues ont édité des textes du Rosarius: aux noms de Zetterberg et de Sandqvist s'ajoute celui d'E. Walberg, dont l'édition Contes pieux en vers français du XIV s. tirés du recueil intitulé Le Tombel de Chartrose, Lund, 1946, est antérieure à la période qui nous concerne.

(51) P. v. Reenen et L. Schøsler, Corpus et stemma en ancien et en moyen français. Bilan, résultats et perspectives des recherches à l'Université libre d'Amsterdam et dans les institutions collaboratrices, in Cl. Buridant (éd.), Le moyen français. Le traitement de texte (édition, apparat critique, glossaire, traitement électronique). Actes du IXe colloque international sur le moyen français, Strasbourg, Presses universitaires de Strasbourg, 2000, pp. 25-54.

(52) Ibidem, pp. 33-34. 


\section{II. Études littéraires}

Les études littéraires, qui forment la deuxième partie de notre présentation, ne nous retiendront pas longtemps. Sauf erreur, pendant la période qui nous intéresse, aucune monographie concernant exclusivement la littérature française entre 1330 et 1500 n'a vu le jour dans les pays nordiques ${ }^{53}$. Cette lacune est un peu contrebalancée par la publication de recueils de contributions à caractère littéraire (cf. ci-dessous).

Deux ouvrages font bande à part dans ce paysage. Le premier est Ogier le Danois dans les littératures européennes de Knud Togeby ${ }^{54}$, vaste étude qui passe en revue la totalité des textes - français, italiens, espagnols, danois et autres - où Ogier joue un rôle. La partie concernant le mfr. se focalise sur les œuvres de Jean d'Outremeuse et de David Aubert, dont les sources ont tout particulièrement retenu l'attention du savant danois. Comme curiosité, signalons qu'un fac-similé de la première édition imprimée du Roman d'Ogier le Danois en prose (Paris, A. Vérard, 1498), est sorti à Copenhague en 1967 avec une introduction du même Togeby ${ }^{55}$. Ce dernier n'est d'ailleurs pas le seul dans les pays nordiques à s'être intéressé à Ogier. Le second volume qui peut être considéré comme une exception positive, Le Moyen Âge par le Moyen Àge, même (v. note 1), abrite en effet un des écrits préparatoires pour l'édition de L'Istoire d'Ogier le redouté (ms. BnF, fr. 1583) de Trond Kruke Saalberg ${ }^{56}$. L'index de ce recueil, divisé en sections selon les genres examinés, illustre bien les centres d'intérêt des chercheurs nordiques: ceux-ci ont contribué aux chapitres concernant les adaptations, les textes épiques et romanesques, mais sont absents des chapitres où il est question de la matière arthurienne et de la poésie lyrique. Sofia Lodén, qui est la seule à s'occuper de traductions/réécritures nordiques de textes français, compare le lion d'Yvain à ceux des versions norroise (İvens saga) et suédoise (Herr Ivan). Elle démontre que le lion décrit par Chrétien a donné naissance à deux figures opposées: İven est accompagné d'un animal «sans parole, volonté ou capacité de penser, de nombreuses fois retiré de l'action», tandis que Herr Ivan est suivi d'une bête anthropomorphisée, curieusement désignée à la fois par le pronom féminin hon ('elle') et le substantif masculin leon (lion et non pas lionne) ${ }^{57}$. Jonna Kjær s'intéresse à une autre réécriture, française cette fois, du Chevalier au lion et se demande si le roman de La Dame à la Licorne peut être considéré comme un modèle d'une maquette de tapisserie ${ }^{58}$. Avec la contribution d'Outi Merisalo, on passe à l'analyse du contexte historique et culturel de la transmission manuscrite du remaniement français du Xv ${ }^{\mathrm{e}}$ siècle de Gui de Warewic ${ }^{59}$.

Les contributions en forme d'articles ou de chapitres de livres portent le plus souvent sur des textes dont l'auteur prépare ou a préparé une édition critique. Ce-

(53) La littérature des XIV et XV siècles n'a cependant pas laissé les chercheurs indifférents. Pour s'en convaincre, il suffit d'évoquer deux titres, La Nouvelle Française au XV siècle, Paris, Champion, 1910, «Bibliothèque du XV siècle», de W. Söderhjelm, et La prose française du XV siècle. Étude esthétique et stylistique, Copenhague, Munksgaard, 1958, de J. Rasmussen.

(54) K. Togeby, Ogier le Danois dans les littératures européennes, Copenhague, Munksgaard, 1969.

(55) Ogier le Dannoys: roman en prose du XV siècle, København, Munksgaard, 1967.

(56) T.K. Saalberg, Prolégomènes à une édition de l'"Istoire d'Ogier le redouté" (BnF fr. 1583). IV: Les sources du récit de la naissance d'Ogier, in Le Moyen Âge... cit., pp. 97-111.

(57) S. Lodén, Le lion d'Yvain revisité: traduire et adapter Chrétien de Troyes dans les pays nordiques, in Le Moyen Âge... cit., pp. 179-194, ici p. 191.

(58) J. Kjær, Réécriture et allégorisation au XIV siècle: l'exemple du "Chevalier au Lion" transformé en "Romans de la Dame à la Lycorne et du Biau Chevalier au Lyon", in Le Moyen Âge... cit., pp. 197-210.

(59) O. Merisalo, La Fortune de Gui de Warewic à la fin du Moyen Âge (XV siècle), in Le Moyen Ầge... cit., pp. 239-253. 
pendant, comme l'a constaté fort judicieusement Mari Bacquin ${ }^{60}$, les philologues nordiques ont tendance à s'intéresser plutôt à l'analyse linguistique des textes qu'à leur aspect littéraire. Bacquin constitue elle-même une exception bienvenue à cette tendance: son édition de Theseus de Cologne, chanson de geste riche en éléments "folkloriques", est précédée d'une longue étude sur les motifs, faite à l'aide d'outils méthodologiques sophistiqués.

Une bonne partie des contributions littéraires nordiques a été publiée dans la "Revue Romane" qui se distinguait naguère encore par l'attention qu'elle portait sur la littérature médiévale. La collaboration assidue de Jonna Kjær y a été pour beaucoup. De ses travaux traitant la littérature en mfr. citons, en guise d'exemple, L'Histoire de la Chastelaine du Vergier et de Tristan le chevalier ${ }^{61}$. Selon l'interprétation proposée par Kjær, cette réécriture de la Chatelaine de Vergi, dont le héros masculin s'appelle d'ailleurs Tristan, pourrait avoir «un rapport avec la cour de Bourgogne du dernier quart du XIV ${ }^{\mathrm{e}}$ siècle» et expliquer «comment les conventions de la cour ont détruit l'amour et, par là, la cour elle-même» ${ }^{62}$. Parmi les contributions de la "Revue Romane", il faut retenir aussi les deux articles dans lesquels le Néerlandais Jelle Koopmans et le Danois Svend Hendrup débattent le problème épineux de la classification des genres du théâtre profane médiéval ${ }^{63}$. En effet, si l'apport des philologues nordiques aux études littéraires reste maigre, cela ne veut nullement dire qu'ils ne s'intéressent pas à ce qui se fait ailleurs dans ce domaine, comme le démontrent les nombreux comptes rendus de J. Kjær, de L. Löfstedt et de S. Sandqvist ${ }^{64}$.

L'auteur médiéval dont le nom apparaît le plus souvent sous la plume de chercheurs venant d'horizons divers est François Villon. C'est peut-être un hasard - mais un hasard significatif - que l'article qui clôt la bibliographie de Togeby soit une longue présentation de l'édition d'A. Henry et de J. Rychner du Testament Villon: ce compte rendu est déjà précédé d'une étude sur La structure des deux testaments de Villon ${ }^{65}$. Togeby ne cache pas son enthousiasme devant l'édition citée et déclare d'emblée: «Voici enfin l'édition du Testament de Villon que nous attendons depuis 500 ans» ${ }^{66}$.

\section{III. Études linguistiques}

Introduction

La présentation même sommaire des études linguistiques relatives à la période du mfr. parues entre 1965 et 2015 dans les pays nordiques est une tâche compliquée. Si, du côté philologique, le panorama est assez homogène, du côté linguistique les méthodes employées et les problèmes abordés présentent en revanche une plus grande diversité. Un clivage fondamental se dessine déjà entre les travaux basés sur

(60) M. Bacquin, Théséus cit., p. 12.

(61) J. Kjær, L'Histoire de la Chastelaine du Vergier et de Tristan le chevalier (XV' siècle). Essai d'interprétation, "Revue Romane" 23, 2, 1988, pp. 260-282.

(62) Ibidem, p. 280.

(63) J. Koopmans, Frère Guillebert: taxinomies et visualisations d'une farce, "Revue Romane" 24, 1, 1989, pp. 49-64; S. Hendrup, Une sottie méconnue: La "Moralité de Chascun, Plusieurs, le Temps-qui-court, le Monde” du manuscrit La Vallière, "Revue Romane” 25, 2, 1990, pp. 331-340.

(64) Les comptes rendus de Kjær ont paru dans "Revue Romane" (25, 1, 1990; 26, 2, 1991; 36, 1, 2001; 37, 1, 2002; 44, 1, 2009) et ceux de Löfstedt surtout dans "Vox Romanica" (par ex. 40, 1981), tandis que Sandström a publié les siens dans "Zeitschrift für romanische Philologie" (par ex. 110, 1994; 111, 1995).

(65) TraLiLi VIII, 1970, pp. 315-323.

(66) K. Togeby, Gloser et commenter "le Testament Villon", "Revue Romane” 10, 1,1975, pp. 192-198 (ici 192). 
des corpus électroniques et ceux dans lesquels quelques textes sont analysés avec des moyens traditionnels.

Dans le présent volet seront d'abord passées en revue les études d'une certaine longueur, monographies, recueils d'articles, etc. Ensuite, dans deux sous-chapitres différents, il sera question de contributions publiées sous forme d'article. Dans le premier, la perspective est diachronique et dans le second, synchronique. Le grand nombre des articles susceptibles d'être mentionnés fait que ceux-ci n'ont pas tous pu être répertoriés et que cette présentation n'aspire donc pas à l'exhaustivité.

\section{Études monographiques; recueils d'articles}

Ce chapitre s'ouvrira, comme il se doit, avec la première monographie consacrée dans les pays nordiques exclusivement au mfr. Il s'agit de la thèse de doctorat d'Ulla Jokinen, Les relatifs en moyen français. Formes et fonctions $\left(1978^{67}\right)$. Cette étude de plus de 400 pages, traitant un sujet sur lequel manquait une vue d'ensemble, arriva à un moment propice: le début des années 1970 avait en effet vu un essor remarquable d'importants travaux sur le mfr. (R. Martin, M. Wilmet, P. Wunderli), et la décennie fut close par la Syntaxe du moyen français de Martin et Wilmet (1980). Les recherches de Jokinen se basent sur un corpus de 65 textes qui s'échelonnent entre 1330 et 1500 . Les ordinateurs personnels n'existaient pas encore à l'époque, et on peut admirer la manipulation d'un corpus de plus de 12000 relatives, transcrites sur des cartes perforées. L'étude de Jokinen a fait l'objet de nombreux comptes rendus, rédigés entre autres par V. Väänänen, G. Price, R. Martin, M. Wilmet et P. Wunderli. Elle a été louée pour la richesse de ses matériaux, la finesse de ses analyses, la somme des renseignements apportés sur l'emploi des relatifs en mfr. Sur le plan descriptif, c'est une réussite certaine. Par contre, ce qui a attiré des critiques, c'est la réflexion théorique et méthodologique, jugée insuffisante, ainsi qu'une approche statistique tenue pour lacunaire. Väänänen constate aussi que «[r] ien n'est dit pour justifier le choix des dates 1330 et 1500 comme limites du moyen français» ${ }^{68}$, tandis que pour Wilmet la période choisie représente une synchronie un peu large. Toutefois, malgré certaines faiblesses, cette thèse demeure un monument incontournable dans les études des relatifs dans l'histoire du français.

Avec La complémentation du verbe en moyen français et en français moderne $e^{69} \mathrm{de}$ Soili Hakulinen, nous avons également affaire à une thèse de doctorat ${ }^{70}$. Celle-ci se situe dans le champ de la linguistique historique, mais sa rédaction a nécessité des compétences dans divers domaines des sciences du langage: linguistique de corpus, linguistique contrastive, philologie du latin et des périodes anciennes du français, théorie grammaticale. Le corpus est constitué du De Consolatione philosophiae de Boèce, de deux traductions de ce texte en mfr. et d'une traduction en frm. La problématique examinée concerne les changements subis par le mode de complémentation des verbes entre le latin tardif d'une part, le mfr. du début, et ensuite de la fin du XIV siècle, d'autre part, et enfin le français du XX $x^{e}$ siècle. L'auteure constate que dès le début de l'afr. le système connaît une profonde restructuration par rapport au latin; quant

(67) U. Jokinen, Les relatifs en moyen français. Formes et fonctions, Helsinki, Annales Academiae Scientiarum Fennicae, 1978, «Dissertationes humanarum litterarum».

(68) V. Väänänen, c.r. dans "Studia neophilologica” 51, 2, 1979, pp. 349-350, ici p. 349.

(69) S. Hakulinen, La complémentation du verbe en moyen français et en français moderne. Étude diachronique sur la base d'un corpus parallèle de traductions. Tampere, 2007, «Acta Universitatis Tamperensis».

(70) Parmi les autres thèses discutées et publiées en Finlande, il y a encore H. Blankenstein, Le subjonctif en moyen français à la lumière d'un corpus de textes des XIV", XV et XVI siècles, Jyväskylä, 2016, «Jyväskylä Studies in Humanities». 
à la période du mfr., elle est qualifiée de deuxième «période de restructuration» ${ }^{71}$. Par complémentation du verbe, Hakulinen entend des constructions comme les suivantes, tirées de La Consolation de Boèce: a) nous dirons que Diex a eternité; b) tu le diras avoir puissance; c) Que dirons nous donques? et d) tu le diras ygal au goupil. Le corpus examiné regroupe les diverses constructions de 54 verbes retenus. La complémentation «propositionnelle», qu'elle soit infinitive ou à verbe fini, constitue l'objet le plus nouveau de la recherche et le chapitre le plus important de la thèse. Il existait peu de travaux portant sur cette question, car on avait en général privilégié la complémentation nominale (complément d'objet direct nominal ou pronominal). L'une des hypothèses testées - et réfutée par Hakulinen - est qu'à cause de l'influence des textes latins, le mfr. des traductions présentait plus de complémentations infinitives que l'afr. et le frm.: cela est contredit par l'analyse des traductions en mfr. Dans son compte rendu sur la thèse, Christiane Marchello-Nizia ${ }^{72}$ constate qu'elle apporte des acquis novateurs autant sur des hypothèses antérieures concernant le français des traductions que sur l'évolution du mode de complémentation. Il est regrettable que la thèse n'ait pas eu un plus grand retentissement international et qu'elle soit très probablement restée inconnue de plusieurs spécialistes de cette période.

Parmi les monographies linguistiques, la plus récente en date est celle de Bengts$\operatorname{son}^{73}$, focalisée sur la proposition participiale détachée, composée d'un participe (présent ou passé) accompagné de son propre sujet. Ces constructions peu étudiées méritaient d'être examinées de plus près, ne serait-ce que parce qu'elles jouissent d'une grande mobilité et sont dépourvues de contraintes syntaxiques structurant le prédicat verbal. Elles constituent un terrain idéal pour caractériser le style personnel d'un auteur ou d'un traducteur. Il s'agit de structures comme celles-ci:

Lequel chastel par tel convenant ainssy rendu, le roy Loys s'en ala d'ilec a

Saint Jehan d'Angeli (...).

Jean de Noyal, Le Miroir historial, Livre X, éd. Förnegård

Et ceste chose ordenee, la dame a tout son ost s'en issy hors du petit port (...)

Chr. de Pisan, Cité des Dames, éd. E. J. Richards

Cette construction, attestée dès l'époque du mfr. ou même avant, connaitra son apogée au milieu du XV siècle pour ensuite baisser en fréquence à la fin du siècle. Bengtsson part de l'ablatif absolu en latin, le plus souvent rendu dans les traductions en afr. et en mfr. par une temporelle. Le point de départ est la comparaison de textes latins et leur traduction en mfr., mais le corpus contient également des textes écrits directement en français. Le français contemporain n'est pas oublié non plus: la construction existe toujours, du moins dans la langue écrite. L'étude de Bengtsson se recommande par son approche innovatrice. Tout au plus pourrait-on lui reprocher de ne pas définir le terme moyen français: parler «du mfr. et du XVI siècle» lui permet d'inclure dans le corpus de «mfr.» même Gargantua (1535).

Quant aux recueils d'articles, U. Jokinen a édité un volume intitulé Approches du moyen français ${ }^{74}$ auquel ont collaboré aussi bien des chercheurs français que fin-

(71) S. Hakulinen, La complémentation cit., p. 7.

(72) Chr. Marchello-Nizia, c.r. dans "Neuphilologische Mitteilungen" CXII, 1, 2011, pp. 120-121.

(73) A. Bengtsson, L'Essor de la proposition participiale en moyen français, Frankfurt a. M., Peter Lang, 2014.

(74) U. Jokinen et P. Sihvonen-Hautecœur (éds), Approches du moyen français, Jyväskylä, 1988, "Studia Philologica Jyväskyläensia”. 
landais. À ce volume s'en est ajouté ensuite un second ${ }^{75}$, conçu selon les mêmes principes. Bien que les deux recueils soient athématiques, s'en dégagent quelques thèmes communs à plus d'un chercheur, comme le syntagme nominal et l'application de la linguistique textuelle aux textes en mfr. Le troisième et dernier recueil d'articles, Aimer, baïr, menacer, flatter... en moyen français ${ }^{76}$, thématique, réunit une partie des communications faites lors du Ve Colloque de l'AIEMF (Helsinki, 2014). Les études, divisées en littéraires, d'une part, et philologiques et linguistiques, de l'autre, témoignent encore du fait que, dans le Nord, celles-ci prévalent sur celles-là.

Dans sa monographie sur sempres, lues, tost, viste et leurs synonymes $\left(\mathrm{XII}^{\mathrm{e}}-\mathrm{XVI}^{\mathrm{e}}\right.$ siècles ${ }^{77}$ ), Lars Lindvall, tout en évitant les termes ancien et moyen français, divise la période examinée en trois parties, dont celle du milieu concerne les XIV et $\mathrm{XV}^{\mathrm{e}}$ siècles. C'est pourtant le $\mathrm{XVI}^{\mathrm{e}}$ siècle qui s'avérera «particulièrement intéressant puisqu'il forme une époque de transition entre l'ancienne langue et le français moderne» ${ }^{78}$. L'analyse à la fois sémantique et syntaxique de ces termes exprimant l'immédiateté, la postérité et la promptitude permet à l'auteur de faire plusieurs rétrodatations.

Pour terminer, mentionnons encore un ouvrage à part dans les études portant sur le mfr. En fait, il ne s'agit pas vraiment d'une étude, mais d'une petite grammaire de la langue des $\mathrm{XIV}^{\mathrm{e}}$ et $\mathrm{XV}^{\mathrm{e}}$ siècles ${ }^{79}$ à l'intention des étudiants de français. L'auteur, Harald Gullichsen, justifie son entreprise par le fait que l'afr. jouit depuis toujours d'un prestige certain dans les études universitaires, tandis que le mfr. est souvent traité en parent pauvre. L'originalité de cette petite morpho-syntaxe réside dans la constante comparaison avec le français contemporain, faite avec de nombreux exemples à l'appui.

\section{Contributions diachroniques}

Lene Schøsler, auteure de nombreuses études diachroniques relatives à l'histoire de la langue française, est un des premiers romanistes nordiques à avoir basé ses recherches sur des corpus électroniques. Le rôle qu'y joue le mfr. varie d'une étude à l'autre selon l'importance que le phénomène étudié revêtit pendant cette période. Nous allons en présenter brièvement quelques-unes qui illustrent certains des centres d'intérêt de Schøsler, comme les verbes support, la grammaticalisation ${ }^{80}$ et la valence verbale. Entre 2003 et 2015, Schøsler a consacré plusieurs études aux verbes support dans l'histoire du français. Dans ce domaine, elle a été précédée par un autre linguiste danois, Michael Herslund, qui quelques années plus tôt s'était déjà occupé des constructions qu'il a appelées prédicats verbo-nominaux ${ }^{81}$. Ces structures, comportant un objet direct, sont du type tenir conseil, faire compaignie ou avoir volonté. Elles se

(75) E. Sakari et H. Häyrynen (éds), Approches du moyen français, t. II, Jyväskylä, 1992, "Studia Philologica Jyväskyläensia”.

(76) J. Härmä et E. Suomela-Härmä (éds), Aimer, bä̈r, menacer, flatter... en moyen français, Paris, Champion, 2017, «Bibliothèque du Xv siècle».

(77) L. Lindvall, Sempres, lues, tost, viste et leurs synonymes. Étude lexicographique d'un groupe de mots dans le français des XII -XVI siècles, Göteborg, Acta Universitatis Gothoburgiensis, 1971, «Romanica gothoburgensia». Le deuxième chapitre, qui porte sur le quatorzième et le quinzième siècles, occupe les pp. 112-143.

(78) Ibidem, p. 16. C'est nous qui soulignons.

(79) H. Gullichsen, Formes et structures du moyen français dans leurs rapports avec la langue contemporaine. Petite grammaire de la langue aux XIV et XV siècles. Oslo, Université d'Oslo [1984] (ronéotypé).

(80) L. Schøsler, Grammaticalisation de phases et d'aspects? À propos des constructions de verbes supports, "Journal of French Language Studies" 25, 2015, pp. 239-263.

(81) M. Herslund, Les prédicats verbo-nominaux en moyen français, in G. Di Stefano et R.M. Bidler (éds), Autour de Jacques Monfrin. Néologie et création verbale, "Le Moyen français" 39-41, 1997, pp. 327-343. 
figent en mfr. et entrent dans le lexique comme locutions verbales, représentant les prédicats verbo-nominaux du titre de l'article. Herslund constate que le mfr. n'est pas une simple phase de transition, mais que c'est la phase de création d'une langue nationale ${ }^{82}$.

Pour en revenir à Schøsler, ses études sur le verbe support se rattachent à d'autres questions essentielles sur lesquelles elle s'est penchée, comme la grammaticalisation et la valence verbale. Une construction à verbe support se développe progressivement à partir de constructions libres composées d'un verbe et de ses compléments, par ex. demander conseil, faire bonne chère, prendre garde, etc. Dès les premiers textes se présentent différents schémas valenciels, comme ceux avec un argument nominal (complément direct) et prépositionnel, qui constitue le noyau lexical (mais il existe aussi des schémas valenciels plus complexes du type demander conseil à qqn, prendre conseil de qqn). En se grammaticalisant, le verbe initialement lexical devient progressivement une sorte d'auxiliaire. Dans les travaux en question, la perspective est souvent très ample, allant de l'afr. au $\mathrm{frm}^{83}$. Le mfr. occupe une place de premier plan par ex. dans l'Étude sur le développement des constructions à verbes supports ${ }^{84}$, où le corpus principal est composé de textes du XIV siècle. Le terme ancienne langue est cependant employé, dans cet article-ci comme ailleurs, pour renvoyer aussi bien à l'ancien qu'au moyen français. La dizaine d'études consacrées par Schøsler à la problématique des verbes support pourrait à juste titre donner lieu à une monographie d'ordre diachronique. La valence verbale joue un rôle de premier plan aussi bien dans Réflexions sur l'optionnalité des compléments d'objet direct ${ }^{85}$ que dans un article datant de $2009^{86}$. Ici aussi Schøsler passe en revue toute l'histoire de la langue française pour examiner l'ellipse de l'objet. Il s'agit donc des rapports entre sujet et objet et de leur rôle changeant dans l'histoire de la langue; parmi les exemples en mfr., citons:

Si regarda la pucelle $(\mathrm{S})$ Cleriadus $(\mathrm{O}) /$

Trestut le pople (S) lodet Deu (O) e _ graciet (Cleriadus)

Ces brefs résumés suffiront à démontrer que la production de Lene Schøsler témoigne d'une grande cohérence dans le choix de ses thèmes de recherche. Avec ses études, elle construit un ensemble remarquable de travaux qui apportent une contribution essentielle à notre connaissance de l'histoire de la langue française.

Des travaux portant sur le système verbal de Jan Lindschouw, nous retenons ici l'article sur l'évolution du système du futur du mfr. au frm. ${ }^{87}$, basé également sur des

(82) Ibidem, p. 340.

(83) Â côté des études allant du latin au frm., dans certains de ses travaux, Schøsler examine des périodes moins longues, notamment l'ancien et le moyen français. Voir par ex. P. van Reenen et L. Schøsler, Ancien et moyen français: si 'thématique'. Analyse exbaustive d'une série de textes, "Vox Romanica" 51, 1992, pp. 101-127 et P. van Reenen et L. Schøsler, La déclinaison en ancien et en moyen français, deux tendances contraires, in Le moyen francais. Philologie et linguistique cit., pp. 595-612.

(84) L. Schøsler, Etude sur le développement des constructions à verbes supports, in B. Fagard et al., éds, Évolutions en français. Études de linguistique diacbronique, Peter Lang, Bern, 2008, pp. 345-361.

(85) L. Schøsler, Réflexions sur l'optionnalité des compléments d'objet direct en latin, en ancien français, en moyen français et en français moderne, in La langue, les signes et les êtres: Actes du colloque de l'Institut d'Études Romanes de l'Université de Copenhague, le 3 octobre 1998, Copenhague, Museum Tusculanum Press, 1999, "Études romanes" pp. 9-28.

(86) L. Schøsler, Les verbes exprimant la notion d'aide, in L. Baronian et F. Martineau (dir.), Le français, d'un continent à l'autre, Mélanges offerts à Yves Charles Morin, Sainte-Foy, Les Presses de l'Université Laval, 2009, pp. 443-465.

(87) J. Lindschouw, L'évolution du système du futur du moyen français au français moderne: la réorganisation comme un cas de régrammation, "Revue de linguistique romane" 75, 2011, pp. 51-97. 
corpus électroniques. L'auteur part de la constatation que le futur synthétique est attesté dès les premiers textes, tandis que les premières occurrences du futur analytique n'apparaissent qu'au milieu du XIV ${ }^{\mathrm{e}}$ siècle. La problématique examinée concerne la réorganisation du système du futur à partir du mfr.; une des conclusions de Lindschouw est qu'en mfr. le futur analytique avait une valeur rétrospective et une valeur prospective, mais que celle-là «a disparu... dans le passage du 17 e au 18 e siècle» ${ }^{88}$.

En se penchant sur la fixation de l'indice de l'infinitif, c'est-à-dire l'élément à ou $d e$ introduisant un syntagme infinitif, Soili Hakulinen reprend une question à laquelle Schøsler s'était déjà intéressée pour l'afr ${ }^{89}$. En interrogeant deux corpus de mfr. dépassant trois millions de mots, Hakulinen démontre que l'emploi de l'indice, sans être totalement aléatoire au XIV ${ }^{\mathrm{e}}$ siècle, obéissait déjà à une certaine logique avec quelques verbes et constructions ${ }^{90}$.

Avant de clore ces remarques, ajoutons encore que dans les années 1980, Ulla Jokinen a fondé à l'Université de Jyväskylä un corpus de textes en moyen français comptant environ un million de mots ${ }^{91}$ qui a rendu des services à plus d'un spécialiste de la période en question.

\section{Contributions synchroniques}

Les contributions retenues ici se laissent regrouper autour de quelques thèmes récurrents. Commençons par les études sur la graphématique de deux chercheurs norvégiens, Lars Otto Grundt et Lise Lorentzen ${ }^{92}$, qui constituent la première tentative d'appliquer ce type d'analyse sur un texte en mfr. Les études en question présentent aussi une autre nouveauté à l'époque, le recours à l'ordinateur, dont les auteurs se sont servis pour relever les autographèmes (la terminologie est celle employée par Paul Diderichsen et Ebbe Spang-Hanssen pour l'analyse du danois écrit ${ }^{93}$ ) dans le Traité de cosmographie de Jean Fusoris (BnF fr. 9558) (ca. 1432). Grundt avait préparé à cette fin une édition «préliminaire» ${ }^{94}$, retouchée ensuite afin de faciliter l'analyse. Grâce à l'ordinateur, Grundt et Lorentzen ont pu, contrairement à leurs prédécesseurs, «examiner tous les mots du corpus, qu'ils soient constitués d'un ou de plusieurs noyaux vocaliques» ${ }^{95}$. Bien que la contribution de 1981 finisse par le souhait que ces recherches soient poursuivies et qu'aux études traditionnelles de graphonomie s'ajoutent des analyses comparative, synchronique et diachronique, l'ouverture de Grundt et Lorentzen ne semble pas avoir eu de suite.

(88) Ibidem, p. 92.

(89) L. Schøsler, Les indices de l'infinitif en ancien français, une étude diachronique, in L. Lindvall (éd.), Actes du $X^{\mathrm{e}}$ Congrès des Romanistes Scandinaves, Lund, Lund University Press, 1990, pp. 414-426; P. van Reenen et L. Schøsler, Les indices d'infinitif complément d'objet en ancien français, in R. Lorenzo (éd.), Actas do XIX Congreso Internacional de Lingüística e Filoloxía Románicas, t. V, A Coruña, Fundación "Pedro Barrié de la Maza, Conde de Fenosa”, 1992, pp. 523-545.

(90) S. Hakulinen, La fixation de l'indice de l'infinitif dans l'évolution du français, in J. Havu et al., (éds), Actes du XVII Congrès des Romanistes Scandinaves, Tampere, Tampere University Press, 2010, pp. 377-395, http://urn.fi/urn:isbn:978-951-44-8339-4.

(91) https://research.csc.fi.

(92) L.-O. Grundt et L. Lorentzen, Essai d'une description graphématique d'un manuscrit français du $X V^{e}$ siècle, in L. Carlsson (éd.), Actes du Ge Congrès des Romanistes Scandinaves, Uppsala, 1977, "Studia Romanica Upsaliensia” pp. 111-124; L. Lorentzen, Étude de graphonomie appliquée à un texte du moyen français, "Revue Romane" 16, 1981, pp. 98-119. Le second article reprend en partie le chapitre rédigé en norvégien par Lorentzen pour l'article paru en 1977.

(93) L.-O. Grundt et L. Lorentzen, Essai d'une description graphématique cit., p. 117.

(94) Cette édition publiée à Bergen en 1973 a disparu même des rayons de la bibliothèque universitaire de Bergen, ville où Grundt a travaillé jusqu'à la retraite. Par conséquent, elle ne figure pas dans la table I.

(95) L. Lorentzen, Étude cit., p. 101. 
À partir des années 1980, les connecteurs et autres particules énonciatives commencent à occuper une place importante dans la linguistique française. Le point de départ des études en question est le français moderne, mais les linguistes ont vite fait d'élargir leur champ d'investigation aux périodes plus anciennes de la langue. On peut citer à ce propos l'étude de Bengtsson sur le connecteur car dans les Chroniques de Froissart et le Brut anglo-normand en prose ${ }^{96}$. Certains lexèmes considérés comme adverbes de temps peuvent aussi jouer un rôle se rapprochant de celui des «particules narratives» ou «marqueurs énonciatifs». C'est ce qui résulte des analyses d'Ellen Sakari97 et d'Olli Välikangas ${ }^{98}$.

Une autre nouveauté dans les études du mfr. pendant la période 1965-2015, c'est qu'on commence à reconnaître que les traductions méritent d'être analysées au même titre que les textes écrits directement en français. Que les traductions et les traducteurs soient désormais appréciés à leur juste valeur, se manifeste aussi bien par le choix des textes à éditer (cf. la table I B ci-dessus) que par les études (pas seulement traductologiques) qui leur sont dédiées ${ }^{99}$. Rares sont cependant les analyses linguistiques publiées en dehors des éditions en préparation ou déjà publiées: à ce propos, on ne peut citer qu'une étude sur la traduction en mfr. de l'ablatif absolu latin ${ }^{100}$ et une autre sur la façon dont Laurent de Premierfait rend en français les noms propres du Décaméron ${ }^{101}$.

Pour passer à des sujets plus traditionnels, les études lexicologiques ne manquent pas, ce dont témoignent les contributions de Leena Löfstedt, connaisseuse des langues de spécialité, comme la terminologie gastronomique ${ }^{102}$. Les termes honorifiques et, plus encore, les termes d'adresse ne cessent d'intéresser les chercheurs finlandais. Pour ce qui est de ceux-là, on peut retenir l'étude de Teija Kähärä sur la concurrence entre sire, seigneur et monseigneur ${ }^{103}$. Les invectives, en l'occurrence celles lancées par des femmes offensées dans la première traduction du Décaméron ${ }^{104}$, sont en quelque sorte le contraire des termes honorifiques. Quant aux termes d'adresse à proprement parler, dans une contribution qui doit beaucoup aux travaux des philologues allemands du début du Xx $x^{e}$ siècle, Juhani Härmä et Elina Suomela-Härmä ont examiné l'alternance émotionnelle et fonctionnelle de Tu et de Vous dans des Mystères

(96) A. Bengtsson, Le rôle du connecteur car (ou nam/enim) dans la prose bistorique: connecteur interphrastique?, "The Medieval Chronicle” XI, 2017, pp. 43-63.

(97) E. Sakari, Observations sur quelques adverbes de temps (or, lors, alors) en moyen français, in B. Combettes et S. Monsonégo (éds), Le moyen français. Philologie et linguistique. Approches du texte et du discours, Actes du VIII ${ }^{\mathrm{C}}$ Colloque international sur le moyen français, Paris, Didier Érudition, 1997, pp. 351-368.

(98) O. Välikangas, La notion de "alors" en mfr., in O. Merisalo et T. Natri (éds), Actes du XIII ${ }^{e}$ Congrès des Romanistes Scandinaves, v. II, Jyväskylä, 1998, «Publications de l'Institut des Langues Romanes et Classiques», pp. 819-826.

(99) E. Suomela-Härmä, Simon Bourgouin, traducteur à l'avant-garde, "Studi francesi" 2/LIX, 2015, pp. $235-246$.

(100) A. Bengtsson, Quelques observations sur la traduction de l'ablatif absolu en moyen français, in Cl. Galderisi et C. Pignatelli (éds), La traduction vers le moyen français. Actes du IIe Colloque de l'AIEMF, Turnhout, Brepols, 2007, «Traduire au Moyen Âge», pp. 205-222.

(101) E. Suomela-Härmä, I nomi propri del "Decameron" nella traduzione di Laurent de Premierfait, in Actes du XVII Congrès cit., pp. 1199-1210, http://urn.fi/urn:isbn:978-951-44-8339-4.

(102) L. Löfstedt, 'Ypocras' et le 'Mestier': Contributions à l'étude du vocabulaire du "Ménagier de Paris", "Neuphilologische Mitteilungen” LXXXII, 1, 1981, pp. 62-65; Du rôt, du poisson doux et des épices, id., LXXXII, 4, 1981, pp. 428-433; À propos du livre de cuisine du Ménagier de Paris, id., CVII, 3, 2006, pp. 323-333.

(103) T. Kähärä, [Le] Nom d'adresse sire en moyen français, in Le moyen français. Philologie et linguistique cit. pp. 281-295.

(104) M. Helkkula, «Villain chien deshonneste!» Sur les invectives proférées par des femmes trahies dans la première traduction française du Décaméron. In Aimer, hä̈r cit., pp. 141-152. 
et autres textes religieux destinés à être joués ${ }^{105}$. Des sujets de recherches ayant attiré l'attention de plus d'un chercheur sont aussi le syntagme nominal ${ }^{106}$ et la réduplication synonymique ${ }^{107}$ dans un texte donné, parfois édité par l'auteur de l'article.

\title{
Conclusion
}

Pendant la période dont nous nous sommes occupés dans les pages qui précèdent, l'étude du mfr. a connu un essor remarquable dans les pays nordiques. Avant les années 1960, les chercheurs nourrissaient un intérêt modeste envers cette période "de transition" et consacraient leur énergie à l'étude de la langue et littérature des XII et XIII ${ }^{\mathrm{e}}$ siècles. Ensuite, leur centre d'intérêt s'est déplacé ou du moins il s'est élargi (le phénomène ne se limite nullement aux pays nordiques, bien au contraire: ceux-ci ne font que suivre des tendances qui se sont développées en France, en Italie, en Allemagne et ailleurs encore). Même sans avoir mené une enquête sur la question, nous ne pensons pas nous tromper en affirmant qu'actuellement les études nordiques sur le mfr. sont devenues quantitativement plus importantes que celles portant sur l'afr. Cependant, certains problèmes persistent; la question qui divise le plus les chercheurs, aussi bien philologues que linguistes, est la délimitation temporelle de la période du mfr. Peut-être faut-il en conclure qu'il n'existe pas de solution satisfaisante à cette question.

Le médiéviste nordique qui s'occupe du mfr. est surtout un éditeur de texte ou un linguiste. Philologue, il publie de préférence des textes religieux, édifiants ou "divulgatifs" (surtout cynégétiques), parmi lesquels les traductions intra- et interlinguistiques ont une place non négligeable. Linguiste, il se concentre soit sur des phénomènes syntaxiques et examine des périodes longues, dépassant le seul mfr. aussi bien en aval qu'en amont, soit il analyse des phénomènes morpho-syntaxiques ponctuels chez un auteur spécifique.

Revenons encore à la constatation de Gaston Paris citée au début de notre tour d'horizon. Comme nous l'avons vu, le savant français commence par mettre en relief la vivacité des études philologiques en Suède; à l'heure actuelle, on n'hésite toujours pas à souscrire à son jugement. Par contre, dans les autres pays nordiques la situation est moins brillante. Les études philologiques y ont cédé le pas à l'étude du français contemporain, de sorte que les médiévistes encore actifs ne peuvent guère y compter sur la jeune génération. Celle-ci ne partage pas ou partage peu leur intérêt pour la philologie et les études diachroniques. Toutefois, cette tendance n'est pas nécessairement irréversible: nous devons maintenant conjuguer nos efforts afin de susciter de nouvelles vocations.

\author{
ELINA SUOMELA-HÄRMÄ \\ Université de Helsinki \\ JUHANI HÄRMÄ \\ Université de Helsinki
}

(105) E. Suomela-Härmä et J. Härmä, Regards sur l'alternance allocutoire en moyen français, in C. Guillot, S. Heiden et S. Prévost (éds), À la quête du sens. Études littéraires, historiques et linguistiques en hommage à Christiane Marchello-Nizia, Lyon, ENS Éditions, 2006, «Langages», pp. 231-243.

(106) U. Jokinen et O. Merisalo, Le syntagme nominal non-déterminé et indéfini pendant la période 1430-1530 du moyen français, in Le moyen français. Philologie et linguistique cit., pp. 507-515; E. Sakari, La détermination du nom en moyen français: l'article défini et ses substituts opposés au marquant zéro dans les "Mémoires" de Philippe de Commynes, in Approches cit., pp. 141-178.

(107) U. Jokinen, Réduplication synonymique dans quelques poèmes politiques de Pierre Gringore, in G. Di Stefano et R. M. Bidler (éds), La grande rhétorique. Hommage à la mémoire de Paul Zumthor, "Le Moyen français" 34, 1994, pp. 103-122. 